\title{
Natural history of innocent heart murmurs in newborn babies: controlled echocardiographic study
}

\author{
Romaine Arlettaz, Nicholas Archer, Andrew R Wilkinson
}

\begin{abstract}
Aim-To define the origin and the natural history of innocent heart murmurs in newborn infants.

Methods-Fifty healthy babies born at term with the clinical diagnosis of an innocent heart murmur and 50 controls without a murmur were studied. Each baby had a complete two dimensional and pulsed Doppler echocardiogram and those with any abnormality were followed up at 6 weeks, 3 months, and 6 months until the murmur had disappeared or the heart was totally normal. Babies with congenital heart disease were excluded from the study.

Results-Pulmonary branch stenosis (PBS) was found in $25(50 \%)$ of the study group and in six (12\%) controls; patent ductus arteriosus (PDA) in $30(60 \%)$ subjects and in six (12\%) controls; and a patent foramen ovale (PFO) in $50(100 \%)$ subjects and in $41(82 \%)$ controls. At 6 weeks the murmur had disappeared in $64 \%$ of babies. PBS was still present in eight of $22(36 \%)$ babies at 6 weeks, in $12 \%$ at 3 months, but in none at 6 months. At 6 weeks, seven of the eight with PBS still had a murmur compared with two of 14 (14\%) babies in which the PBS had resolved $(P<0.005)$. At 6 weeks the PDA had closed in all patients but the foramen ovale was still patent in 29 of $44(66 \%)$ subjects and nine of $33(27 \%)$ controls. The closure of the PFO was not influenced by its size at birth. Conclusions-An innocent heart murmur in a baby born at term is often related to PBS, particularly if the murmur is still present after 24 hours of age, when most PDA have closed. At 6 weeks the murmur had disappeared and the PBS had resolved in $64 \%$ of the babies. PBS had resolved in all babies at 6 months.
\end{abstract} (Arch Dis Child Fetal Neonatal Ed 1998;78:F166-F170)

Neonatal Unit Department of Paediatrics

University of Oxford John Radcliffe Hospital Oxford OX3 9DU

Correspondence to: Professor Wilkinson.

Accepted 1 December 1997 latory changes occurring after birth, the exact cause of these murmurs has not been established. The closure of the foramen ovale ${ }^{23}$ and of the ductus arteriosus $s^{4-6}$ have been the subject of many studies. However, a murmur thought to be of ductal origin is described in only $14 \%$ of healthy babies ${ }^{1}$ and the patent foramen ovale is not usually detectable clinically. Most babies presenting with an innocent heart murmur have a mid systolic ejection murmur which is of grade $1-2 / 6$ in intensity, located in the pulmonary area, radiates to the axillae and the back, and typically disappears by 3-6 months of age. All of which suggests that the origin is in a branch of the pulmonary artery. This hypothesis is supported by clinical ${ }^{7}$ and angiographic ${ }^{8}$ studies. However, echocardiographic studies of newborn babies with an innocent heart murmur in the first days of life have not been reported.

This study aimed to: (i) define the echocardiographic features of newborn babies with a heart murmur considered clinically to be innocent; (ii) confirm the hypothesis that an innocent heart murmur in the neonatal period frequently has its origin in a pulmonary artery branch; and (iii) define the time of disappearance of the murmur.

\section{Methods}

All healthy term newborn babies born in the John Radcliffe Hospital, Oxford, over five months, and who were thought to have an innocent heart murmur at the time of the initial full examination, were studied. After the murmur was first detected on routine examination the clinical innocence of the murmur was confirmed by one investigator (RA).

During the same period, an equal number of babies without a heart murmur were selected as a control group and examined in the same way. Each baby had a complete M-Mode, two dimensional, and pulsed Doppler echocardiogram. The babies were studied using either an ATL Ultramark 4 scanner with a $7.5 \mathrm{mHz}$ transducer, or a $5 \mathrm{mHz}$ Doppler crystal, or with an ATL Apogee CX 3.2 scanner with a 7.5 $\mathrm{mHz}$ transducer, with pulsed and continuous wave Doppler. The procedure for the echocardiogram was as follows: structural heart disease was excluded, then the aortic valve was visualised from the parasternal long axis view and the 
Table 1 Characteristics of the newborn infants

\begin{tabular}{llll}
\hline & Study group $(n=50)$ & Control group $(n=50)$ & $p$ Value \\
\hline Male:female & $24: 26$ & $25: 25$ & NS \\
Gestational age (weeks) & $39.8(1.1 \mathrm{SD})$ & $39.4(1.3 \mathrm{SD})$ & $\mathrm{NS}$ \\
Range & $37-42$ & $37-42$ & \\
Birthweight (g) & $3744(626 \mathrm{SD})$ & $3375(546 \mathrm{SD})$ & $<0.01$ \\
Range & $2725-6239$ & $2124-4709$ & $<0.001$ \\
Age at echo (hours) & $44(50 \mathrm{SD})$ & $57(37 \mathrm{SD})$ & \\
Range & $6-252$ & $10-184$ & \\
\hline
\end{tabular}

internal diameter between hinge points of the valve cusps measured with the valve at its maximal opening. The pulmonary artery and its branches were visualised from the parasternal short axis view. The internal diameter of the pulmonary valve was similarly measured at the time of maximal opening of the valve. The internal diameters of the right and the left pulmonary arteries were measured at their origin. An M-Mode study of the left ventricle from the parasternal long axis view was used to measure the left ventricular dimensions and shortening fraction. From the parasternal short axis view, the pulsed Doppler sample volume was placed distal to the pulmonary valve to measure the pulmonary artery blood flow velocity. For each velocity, a mean of three measurements was taken. The sample volume was then displaced to the origin of the right and the left pulmonary branches to measure velocity. The aortic arch was visualised from the suprasternal notch and the sample volume placed distal to the aortic valve to measure velocity in the ascending aorta and distal to the isthmus to measure velocity in the descending aorta. The ductus arteriosus was then visualised from the high parasternal view and when possible the flow pattern was recorded. Finally, the atrial septum was visualised from the subcostal view and the sample volume was placed at the level of the fossa ovalis to measure the maximal velocity through the foramen ovale.

Follow up occurred at the age of 6 weeks, 3 months, and 6 months unless the heart murmur had disappeared or the echocardiogram was totally normal. The control babies had no follow up unless a patent ductus arteriosus (PDA), a patent foramen ovale (PFO), or pulmonary branch stenosis (PBS) was detected. We defined PBS on echocardiogram as an increase of the peak velocities of at least $50 \%$ between the main pulmonary artery and one or both branches. ${ }^{9}$ If only a PFO had been detected, the babies in both groups were discharged at 6 weeks if the foramen ovale was $2 \mathrm{~mm}$ or less in diameter. This decision was made following the work of Radzik et al which showed that an atrial septal defect smaller than $3 \mathrm{~mm}$ in the neonatal period closes spontaneously in all cases before 9 months of age. ${ }^{10}$

Table 2 Occurrence of patent foramen ovale and patent ductus arteriosus

\begin{tabular}{llll}
\hline & Study group $(n=50)$ & Control group $(n=50)$ & $p$ Value \\
\hline Patent foramen ovale & $50(100 \%)$ & $41(82 \%)$ & $<0.005$ \\
Patent ductus arteriosus & $30(60 \%)$ & $6(12 \%)$ & $<0.0001$ \\
Age $\leqslant 24 \mathrm{~h}$ & $19 / 21(90 \%)$ & $4 / 5(80 \%)$ & $\mathrm{NS}$ \\
Age $>24 \mathrm{~h}$ & $11 / 29(38 \%)$ & $2 / 45(4 \%)$ & $<0.001$ \\
\hline
\end{tabular}

The babies were not sedated for the study. Babies with any respiratory distress were not entered into the study, and those with a structural heart abnormality were excluded. The study was approved by the Central Oxford Research Ethics Committee. Informed written consent from one or both parents was obtained.

Values are presented as median (range). Median values were compared using an unpaired Mann-Whitney U test. Discontinuous variables were compared using the Fisher's exact test. P values of $<0.05$ were accepted as significant.

\section{Results}

INITION EVALUATION

We studied 50 healthy babies born at term with a murmur considered clinically to be innocent. The prevalence of innocent heart murmurs for our hospital was 21 per 1000 live births. Eleven babies with murmurs were excluded from the study because the murmur was considered clinically to be of organic origin: nine had a muscular ventricular septal defect, one had a large secundum atrial septal defect, and one had a tetralogy of Fallot. Table 1 gives the characteristics of the babies in each group. The gestational age was similar in both groups. The babies in the study group were heavier than the controls $(p<0.01)$. The mean age at which the first echocardiogram was performed in the study group was earlier than in the control group so that babies could be discharged from hospital if the murmur was innocent. Twenty one babies in the study group (42\%) and five in the control group (10\%) were less than 1 day old $(\mathrm{p}<0.001)$.

\section{Patent foramen ovale}

All study group babies and $82 \%$ of controls had a PFO; p $<0.005$ (table 2). The mean peak velocity across the foramen ovale was $47.1 \mathrm{~cm} / \mathrm{s}$ in the study group and $41.4 \mathrm{~cm} / \mathrm{s}$ in the control group (NS). The size of the PFO was $>2 \mathrm{~mm}$ in $(36 \%)$ of the 42 subjects and in six $(17 \%)$ of the 35 controls in whom it could be measured.

\section{Patent ductus arteriosus}

Sixty per cent of the study group and $12 \%$ of the controls had a PDA; $\mathrm{p}<0.0001$ (table 2). The mean peak velocity across the ductus arteriosus was $166 \mathrm{~cm} / \mathrm{s}$ in the study group and 159 $\mathrm{cm} / \mathrm{s}$ among the controls (NS). Nineteen of 21 study group (90\%) vs $4 / 5$ controls (80\%) younger than 24 hours had a PDA (NS). Eleven of the 29 in the study group (38\%) vs two out of $45(4.5 \%)$ controls older than 24 hours at initial echocardiogram had a PDA $(\mathrm{p}<0.001)$. No PDA was detected beyond 46 hours of age.

\section{Pulmonary branch stenosis}

The dimensions of the main pulmonary artery and its branches did not show any differences between the groups (table 3). Peak velocity in the main pulmonary artery was similar in both groups. However, the velocity across the pulmonary artery branches was higher in the study group $(\mathrm{p}<0.005)$. The mean increase in 
Table 3 Dimensions and peak velocities across main pulmonary artery and its branches

\begin{tabular}{|c|c|c|c|c|c|c|c|c|c|}
\hline & \multicolumn{3}{|c|}{ Main pulmonary artery } & \multicolumn{3}{|c|}{ Right pulmonary artery } & \multicolumn{3}{|c|}{ Left pulmonary artery } \\
\hline & Study & Controls & $p$ Value & Study & Controls & $p$ Value & Study & Controls & $p$ Value \\
\hline Dimensions (mm) & $9.9(1.4)$ & $9.5(1.2)$ & NS & $4.7(0.7)$ & $4.5(0.8)$ & NS & $4.5(0.9)$ & $4.4(0.7)$ & NS \\
\hline Range & $7-13$ & $7-12$ & & $3-7$ & $3-7$ & & & & \\
\hline Velocity $(\mathrm{cm} / \mathrm{s})$ & $81.9(12.1)$ & $79.3(12.4)$ & NS & $107.5(24.7)$ & $89.3(20.5)$ & $<0.0001$ & $107.8(28.4)$ & $91.0(22.9)$ & $<0.005$ \\
\hline Range & $58-113$ & 58-106 & & 70-199 & $60-160$ & & $50-180$ & $60-158$ & \\
\hline
\end{tabular}

velocity between the main pulmonary artery and its branches was significantly higher in the study group compared with the control group (table 4). Twenty five babies in the study group $(50 \%)$ compared with six in the control group $(12 \%)$ had an increase of more than $50 \%$ between the main pulmonary artery and one or both branches $(p<0.0001)$. No significant difference was observed between the right and the left pulmonary artery.

Association of several lesions

No baby in the study group, but nine in the control group (18\%) had a completely normal echocardiogram. The overall incidence of several abnormalities in the same baby was greater in the study group than in the control group. Fifteen babies with an innocent murmur had PBS associated with PDA and PFO, compared with two babies in the control group $(\mathrm{p}<0.001)$. PDA as an isolated lesion was not found in either group.

FOLLOW UP

Follow up was performed at the age of 6 weeks, 3 months, and 6 months. In the study group 44 babies were seen at 6 weeks, 15 at 3 months, and 3 at 6 months. In the control group 33 babies were seen at 6 weeks and six at 3 months. All the babies who had missed the 6 week appointment ( $\mathrm{n}=11$; six study group, five

Table 4 Increase in velocity between main pulmonary artery and its branches

\begin{tabular}{llll}
\hline & $\begin{array}{l}\text { Study } \\
\text { group }\end{array}$ & $\begin{array}{l}\text { Control } \\
\text { group }\end{array}$ & p Value \\
\hline $\begin{array}{l}\text { Percentage increase in } \\
\text { velocity between MPA and }\end{array}$ & & & \\
$\begin{array}{l}\text { the branches }( \pm \text { SD) } \\
\text { Range }\end{array}$ & $\begin{array}{l}37.0(42) \\
0-210\end{array}$ & $\begin{array}{l}19.5(38) \\
0-200\end{array}$ & $<0.001$ \\
\hline
\end{tabular}

Table 5

\begin{tabular}{lll}
\hline & Follow up & \\
\cline { 2 - 3 } & Study group & Control group \\
\hline First study & $\mathrm{n}=50$ & $\mathrm{n}=50$ \\
& & 9 normal scan \\
6 weeks & $\mathrm{n}=44$ & $\mathrm{n}=33$ \\
& 28 no murmur & 1 murmur, normal \\
& 7 murmur, normal & scan \\
& scan & 3 months \\
& 6 DNA (all seen at & \\
& 3 months) & $\mathrm{n}=6$ \\
3 months & $\mathrm{n}=15$ & 5 no murmur, \\
& 7 no murmur & normal scan \\
& & 1 murmur, normal \\
& 5 murmur, normal & scan \\
6 months & scan & $\mathrm{n}=3$ \\
& 2 no murmur & \\
& 1 murmur, normal & \\
& scan & \\
\hline
\end{tabular}

DNA=did not attend. controls) attended at 3 months. The auscultatory characteristics of the babies are shown in table 5 . In the study group 16 of $44(36 \%)$ babies still had a murmur at 6 weeks, eight of $50(16 \%)$ at 3 months, and one of 50 at 6 months. In other words the murmur had resolved in $64 \%$ babies at 6 weeks, in $84 \%$ at 3 months, and in $98 \%$ at 6 months. In the control group one baby presented at 6 weeks with an innocent murmur and another one who had not attended the 6 week follow up presented at 3 months with an innocent heart murmur and a normal heart. One baby included in the study group with PBS at birth later developed a mild valvar pulmonary stenosis, which although not apparent on pulmonary artery Doppler assessment at birth, was found at 6 months with a harsh systolic murmur.

\section{Patent foramen ovale}

All study group babies had a PFO at first assessment: nine had a PFO alone; the others had a PFO associated with PDA ( $=13)$, PBS $(n=7)$, or both $(n=15)$. At 6 weeks, a PFO was still present in 29 of 44 babies (66\%). Fifteen of them still had a murmur $(52 \%)$ and 14 did not. Timing of closure of the PFO was not predicted by its size at birth: at 6 weeks $36 \%$ of the $>2 \mathrm{~mm}$ PFO (eight of 22 ) and $36 \%$ of the 1-2 mm PFOs (five of 14) had closed (NS). No PFO of $3 \mathrm{~mm}$ or more was found. At 3 months, three babies still had a PFO, of 1-2 $\mathrm{mm}$.

In the control group 33 babies had a PFO at first assessment: 26 had a PFO alone; the others had PFO associated with PDA $(n=3)$, PBS $(n=3)$, or both $(n=1)$. At 6 weeks, PFO was still present in nine of $33(27 \%)$ babies. One of the babies, who did not have a murmur at birth, had a murmur at 6 weeks. Seventeen of the $23(74 \%)$ of the PFO of $1-2 \mathrm{~mm}$ in size and $(66 \%)$ of the PFOs $>2 \mathrm{~mm}$ had closed (NS). Again, no PFO of $3 \mathrm{~mm}$ or more was found at 6 weeks. At 3 months, a PFO was detected in only one baby, who had no murmur.

PATENT DUCTUS ARTERIOSUS

All babies with a PDA in both groups were followed up, and no PDA was seen at 6 weeks or at 3 months.

\section{Pulmonary branch stenosis}

In the study group, 22 followed up babies had PBS at first assessment, which was associated either with PFO $(n=7)$ or with PFO and PDA $(n=15)$. At 6 weeks, eight of $/ 22$ babies $(36 \%)$ still had PBS. Seven had a murmur, compared with two of the 14 babies (14\%) in whom the PBS resolved: this difference is significant $(p<0.005)$. Of these eight babies, one had PBS of the right pulmonary artery and seven had a 
PBS of the left pulmonary artery. At 3 months, PBS was detected in three of these eight babies ( $12 \%$ of the total); two of them had still a murmur. The PBS involved the left pulmonary artery in all three cases. At 6 months the PBS had resolved.

In the control group four babies had PBS at birth: three were associated with $\mathrm{PFO}$ and one with PFO and PDA. At 6 weeks, two of them still had PBS. They did not have a murmur. No control baby had PBS at 3 months.

Normal heart

At birth, no baby in the study group had an entirely normal heart. At 6 weeks, 13 of 44 $(29 \%)$ had a normal heart, and none of these 13 babies had a murmur. At 3 months, 11 more babies had a normal heart on echocardiogram, but five of them still had a murmur.

In the control group nine babies had an entirely normal heart at birth and were not followed up. Thirty three were and 22 of them had a normal heart at 6 weeks. None of these 22 babies had a murmur. At 3 months, five more babies had a normal heart; one of them had an innocent murmur.

\section{Discussion}

Our results suggest that innocent heart murmurs in healthy babies born at term are mostly related to pulmonary branch stenosis, which resolves in two thirds by 6 weeks and in all by 6 months.

Although the concept of physiological PBS was well defined by Danilowicz et al 25 years ago, ${ }^{8}$ few studies have been carried out using echocardiography. Rodriguez et $a l^{10}$ studied preterm infants with a clinical diagnosis of PBS who had small pulmonary branches on echocardiography in which there were high Doppler velocities. Chatelain et $a l^{11}$ studied 21 babies, six of whom were preterm, with similar echocardiographic findings. In their study the PBS had resolved in $64 \%$ at 3 months of age, with a normal echocardiogram and no murmur.

A precise definition of physiological PBS has not been agreed. Clinically, PBS is suspected by the presence of a murmur located in the pulmonary area and radiating widely to the axillae and back. ${ }^{10} 11$ In our study such a murmur was heard in 27 babies; 17 of them had PBS as defined by a $50 \%$ increase in velocity between the main pulmonary artery and its branches. In 23 babies auscultation was not suggestive of PBS, but eight had PBS on echocardiogram. This shows the limitation of clinical examination (sensitivity $68 \%$, specificity $60 \%$, positive predictive value $63 \%$, negative predictive value $65 \%$ ).

In our study the size of the pulmonary arteries was not significantly different between the study and control groups. In the control group the mean diameter was $4.5 \mathrm{~mm}$ for the right and $4.4 \mathrm{~mm}$ for the left, which can be considered to be normal. Only a few studies give normal values which makes comparison difficult. Rodriguez et $a l^{10}$ gives a mean diameter of 5 $\mathrm{mm}$ for the right pulmonary artery and $4.9 \mathrm{~mm}$ for the left, while Chatelain et $a l^{11}$ report a mean diameter of $4.8 \mathrm{~mm}$ for the right and $4.9 \mathrm{~mm}$ for the left pulmonary artery. Snider $e t a l^{12}$ and Lappen $e t ~ a l^{13}$ published graphs of normal values for the size of the right pulmonary artery from newborn to adults, divided into subgroups defined by their body surface area. In both these studies the number of cases was small and the range wide. Lappen ${ }^{13}$ gave larger sizes for the pulmonary artery branches than ours but he used the suprasternal coronal view and the leading edge method, which can explain this discrepancy. The scanners in use make measurements in whole millimetres, not in fractions of a millimetre, and therefore the resolution may be insufficient to detect small differences in the diameter of the pulmonary branches.

We suggest, like many authors, ${ }^{7-9} 1112$ that the relative hypoplasia of the pulmonary artery branches, which are associated with an angulation at their origin that increases velocity and turbulent flow, are responsible for the murmur.

In the fetus there is a continuation between the pulmonary trunk, the large ductus arteriosus, and the descending aorta. As a consequence of the low pulmonary blood flow (about $10 \%$ of the combined ventricular output at term), the pulmonary artery branches remain small and hypoplastic. At birth, there is a marked discrepancy between the large pulmonary trunk and the relatively underdeveloped branches, which increases the blood flow velocity between them. This is amplified by the increase in pulmonary blood flow which occurs as pulmonary vascular resistance decreases. Furthermore, anatomical studies have shown an angulation at the origin of the pulmonary artery branches, causing a pressure difference which could explain the murmur. $^{811}$

Dunkle $e t a l^{7}$ found that the murmur of PBS was present at birth in term but not preterm infants. In the latter it appeared within the first 2 weeks of life and usually disappeared by the age of 6 weeks. The term infants were not followed up. Chatelain et $a l^{11}$ diagnosed PBS on echocardiogram at a mean postnatal age of 23 days, but did not specify the time of appearance of the murmur. As our study was of those babies with an innocent murmur at the first examination, and they were usually discharged home 2 or 3 days after birth, we do not know if a murmur developed in the controls at a later stage. However, only one baby with no PBS at birth had it at 6 weeks. In $64 \%$ of those with PBS it had resolved by 6 weeks, in $88 \%$ by 3 months, and all of them by 6 months. These results are similar to those of Chatelain et al. ${ }^{11}$ We found a good correlation between PBS and a murmur: at 6 weeks $87 \%$ of babies with a PBS had a murmur as well, and only $14 \%$ in whom the PBS had disappeared still had a murmur $(\mathrm{p}<0.005)$.

In the babies under 24 hours of age the incidence of PDA was similar in both groups $(90 \%$ and $80 \%$ ) (table 2). The relation between PDA and a murmur is difficult to establish because every baby in both groups with PDA had another associated abnormality, either PBS, or PFO, or both. However, daily examination and 
an echocardiogram to assess if closure of the ductus arteriosus correlated with disappearance of the murmur was not performed as the babies were discharged home. We examined them 6 weeks later and the PDA had closed in all cases. Gentile $e t a l^{4}$ found a murmur in only $28 \%$ of babies with a PDA confirmed on echocardiogram.

All babies with murmurs and $82 \%$ of controls had PFO at the time of the first study. This agrees with published findings, ${ }^{2} 310$ although there is a wide range. Echocardiograms which did not show $\mathrm{PFO}$ in controls increased from $0 \%$ on day 1 to $30 \%$ on day 4 . No baby with a murmur had a closed foramen ovale. In both groups the foramen ovale was always patent if PBS or PDA was present. The finding that PDA closes before PFO supports that of Hiraishi et $a l^{2}{ }^{2}$

Some authors ${ }^{811}$ suggest that a left to right shunt across the foramen ovale can increase the velocity between the main pulmonary artery and its branches, by increasing pulmonary blood flow. This hypothesis is difficult to confirm: all babies with PBS had a PFO at birth, but all the babies without PBS in the study group and $79 \%$ in the control group had a PFO as well.

It is important to mention that flow across the foramen ovale can be detected after several weeks or several months. In our study two thirds of the study group and a quarter of the controls had PFO at 6 weeks. This is not surprising as Hagen et $a l^{14}$ and Fisher et $a l^{15}$ report an incidence of $\mathrm{PFO}$ in adults of $27 \%$ and $9 \%$, respectively.

Finally, five babies in the study group with a normal heart on echocardiogram still had a murmur at 3 months. Two had PFO at birth, one associated with a PDA, and three had PBS which was present at 6 weeks but which had resolved at 3 months. In these the character of the murmur had changed - at birth it suggested PBS, but at 3 months it was $1 / 6$ mid-systolic at the left lower sternal edge with no radiation.

1 Braudo M, Rowe RD. Auscultation of the heart - early neonatal period. Am f Dis Child 1961;101:575-86.

2 Hiraishi S, Agata Y, Saito K, et al. Interatrial shunt flow profiles in newborn infants: a colour flow and pulsed Doppler echocardiographic study. Heart 1991;65:41-5.

3 Hannu H, Pentti K, Henrik E, Markku S, Ilkka V. Patency of foramen ovale - does it influence haemodynamics in newborn infants? Early Hum Dev 1989;20:281-7.

4 Gentile R, Stevenson G, Dooley T, Franklin D, Kawabori I, Pearlman A. Pulsed Doppler echocardiographic determination of time of ductal closure in normal newborn infants. F Pediatr 1981;98:443-8.

5 Evans NJ, Archer LNJ. Postnatal circulatory adaptation in healthy term and preterm neonates. Arch Dis Child 1990;65:24-6.

6 Reller MD, Rice MJ, McDonald RW. Review of studies evaluating ductal patency in the premature infant. $\mathcal{f}$ Pediatr 1993;122:S59-62.

7 Dunkle LM, Rowe RD. Transient murmur simulating pulmonary artery stenosis in premature infants. Am $\mathcal{F} D$ is pulmonary artery steno

8 Danilowicz DA, Rudolph AM, Hoffman JIE, Heymann M. Physiologic pressure differences between main and branch pulmonary arteries in infants. Circulation 1972;XLV:41019.

9 Radzik D, Davignon A, van Doesburg N, Fournier A, Marchand T, Ducharme G. Predictive factors for spontaneous closure of atrial septal defects diagnosed in the first 3 months of life. 7 Am Coll Cardiol 1993;22:851-3.

10 Rodriguez RJ, Riggs TW. Physiologic peripheral pulmonic stenosis in infancy. Am $\mathcal{f}$ Cardiol 1990;66:1478-81.

11 Chatelain P, Oberhaensli I, Friedli B. Physiological pulmonary branch stenosis in newborns: 2D-echocardiographic nary branch stenosis in newborns: 2D-echocardiographic 1993;152:559-63.

12 Snider AR, Enderlein MA, Teitel DF, Juster RP. Twodimensional echocardiographic determination of aortic and pulmonary artery sizes from infancy to adulthood in normal subjects. Am $\mathcal{F}$ Cardiol 1984;53:218-24.

13 Lappen RS, Riggs TW, Lapin GD, Paul MH, Muster AJ. Two-dimensionalechocardiographic measurement of right pulmonary artery diameter in infants and children. $\mathcal{F} \mathrm{Am}$ Coll Cardiol 1983;2:121-6.

14 Hagen PT, Scholz DG, Edwards WD. Incidence and size of patent foramen ovale during the first 10 days of life: an
autopsy study of 965 normal hearts. Mayo Clin Proc autopsy study

15 Fisher DC, Fisher EA, Budd JH, Rosen SE, Goldman ME. The incidence of patent foramen ovale in 1000 consecutive patients. Chest 1995;107:1504-9. 